\title{
Trayectorias escolares de estudiantes con discapacidad en la educación secundaria argentina: biografías en contexto
}

\author{
School trajectories of students with disabilities at the Argentinian \\ secondary school level: Biographies in context
}

\begin{abstract}
Resumen
La tesis que se presenta en este artículo tiene como objetivo analizar las trayectorias escolares de estudiantes con discapacidad que actualmente cursan el nivel secundario en Argentina. Se considera necesario abordar esa dimensión vital desde un enfoque biográfico narrativo porque permite desentrañar los sentidos profundos de los logros, obstáculos y desafíos que se presentan. Las particularidades de estas trayectorias se vinculan a las derivaciones, los cambios de escuelas, las configuraciones de apoyo y otras dinámicas singulares que son vividas por cada estudiante, según las condiciones y oportunidades de cada contexto. La intención es aportar a los estudios críticos en discapacidad en América Latina, sistematizando las valiosas experiencias que se construyen en nuestros países, desde el respeto por la palabra de los estudiantes con discapacidad, que ha sido históricamente invisibilizada en los contextos académicos.
\end{abstract}

\section{Palabras clave}

Educación, discapacidad, trayectorias escolares, escuela secundaria.

\begin{abstract}
The objective of this thesis presented in this article, is to analyze the school trajectories of students with disabilities currently attending the secondary school level. It is considered necessary to address this vital dimension from a narrative-biographical approach because it allows the unraveling of the deep meanings of the achievements, obstacles and challenges that are to be faced. The distinctive features of these trajectories are linked to the referrals, changes of schools, support systems and other unique dynamics experienced by each student in accordance with the conditions and opportunities each context offers. The intention is to contribute to critical disability studies in Latin America, by systematizing the valuable experiences put together in our countries, respectfully taking into account the voice of students with disability, which has historically been invisible in most academic contexts.
\end{abstract}

\section{Keywords}

Education, disability, school trajectories, secondary school.

\section{Lelia Schewe}

<lelia.schewe@gmail.com>

Universidad Nacional de Misiones. Argentina

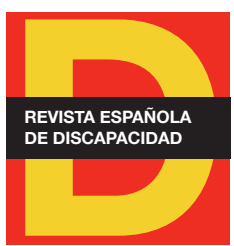

Para citar:

Schewe, L. (2021). Trayectorias escolares de estudiantes con discapacidad en la educación secundaria argentina: biografías en contexto. Revista Española de Discapacidad, 9(2), pp. 103-118.

Doi: <https://doi.org/10.5569/23405104.09.02.06>

Fecha de recepción: 15-04-2021 Fecha de aceptación: 09-12-2021 


\section{Introducción}

Las trayectorias escolares de estudiantes con discapacidad se han reconfigurado durante los últimos 15 años a partir de múltiples procesos, como aquellos vinculados a avances normativos y reconfiguración de ciertas prácticas. Antes de ese lapso temporal, en Argentina y varios países de Latinoamérica era poco probable -en la mayoría de los espacios- la posibilidad de encuentro entre la educación especial y la educación regular para que los estudiantes pudieran elegir a qué tipo de institución deseaban asistir, porque el trabajo en las escuelas de educación especial estaba centrado en garantizar las condiciones necesarias para que quienes asistían a esas escuelas -específicamente- encontraran posibilidades de continuidad. En varios casos, los intentos de intercambios entre escuelas a fin de lo que se denominaba "integración" de estudiantes con discapacidad, fueron entendidos como arriesgados porque las normativas vigentes no los permitían y se consideraban parte de lo que se denominaba "currículum nulo"1.

En el caso de Argentina, la Ley de Educación Nacional ( $N^{\circ}$ 26.206) marcó el inicio de una nueva forma de concebir la educación de estudiantes con discapacidad, generando diversos procesos como la incorporación de resoluciones normativas específicas (como las 174 y la 311 del Consejo Federal de Educación) para que se garantizara el derecho a la educación de este colectivo.

La educación especial continúa siendo el campo de saberes (y la modalidad del sistema educativo, destinada a asegurar el derecho a la educación de estudiantes con discapacidad, como lo define la Ley de Educación Nacional mencionada) que se ocupa de cuestiones vinculadas a la discapacidad, relacionándose formalmente con escuelas de otras modalidades, escuelas regulares y otros espacios educativos. En ese sentido, se consideran fundamentales las discusiones actuales sobre la educación inclusiva, la continuidad de las escuelas de educación especial como espacios específicos (Schewe, 2011, 2021), los recursos necesarios para el trabajo docente, las condiciones de los acompañamientos y los apoyos.

Las trayectorias escolares han sido analizadas como los recorridos que los estudiantes realizan durante ese transitar (Terigi, 2007), en todos los niveles y modalidades del sistema educativo. En el caso de los estudiantes con discapacidad, se presentan particularidades con respecto a las configuraciones y oportunidades de continuidad, que es necesario señalar. Por ejemplo, si un estudiante concurre a una escuela regular, encuentra los apoyos necesarios desde el nivel inicial y obtiene las certificaciones correspondientes, tiene posibilidades de transitar hacia los niveles siguientes, hasta llegar al nivel superior². En cambio, si un estudiante realiza su escolaridad primaria en instituciones de modalidad de educación especial, para que sea posible la transición al nivel siguiente necesita de una serie de procesos particulares. En este sentido, se entrecruzan las trayectorias escolares con transformaciones históricas, políticas e incluso ideológicas con respecto a la discapacidad, trascendiendo las cuestiones curriculares (Briscioli, 2013).

La construcción histórica de las dinámicas que se mencionan, en el sistema educativo argentino, estuvo marcada por la "derivación" (Schewe, 2020) de los estudiantes con discapacidad a espacios particulares para su atención -las escuelas o centros de educación especial- por lo que las continuidades que se analizan en este trabajo constituyen un cambio trascendental. En este sentido, la escuela secundaria estaba inicialmente dirigida a un perfil de estudiante, a aquel que se mantuviera dentro de ciertos parámetros, de

1. Se puede observar algunas de estas dinámicas en https://bdigital.uncu.edu.ar/objetos_digitales/3948/scheweruedes2.pdf.

2. Esos niveles corresponden en Argentina a: inicial, primaria, secundaria y superior. 
cierta norma. Como toda norma es parte de vínculos de poder e implica clasificaciones y -en este caso- un principio de corrección (Pérez, 2014; Cheli, 2013; Vain, 2009), los estudiantes que acceden a estos procesos son solamente quienes cumplen con determinados requisitos establecidos por las instituciones y por los equipos de apoyo. En estos espacios, además, aparecen como parte de las complejidades las concepciones sobre la cuestión de la discapacidad de quienes los integran.

Autores como Danel (2018) consideran que la construcción del concepto discapacidad se vincula a discusiones sobre sus contextos y formas de reproducción: si se considera que se trata de una cuestión social o cultural, el eje se pondrá en elementos y configuraciones de esos órdenes, lo que significa una diferencia sustancial con los modelos impuestos por los paradigmas vinculados a la medicina y al asistencialismo que atraviesan la historia de la discapacidad. En este caso, como se manifiesta a lo largo del trabajo, consideramos que la discapacidad es una experiencia (Torres et al., 2019) y que no se puede (o resultaría absolutamente sesgado, insuficiente y reduccionista) definir solamente desde la perspectiva de organizaciones internacionales o disciplinas específicas vinculadas a la salud, a la educación, o desde el campo del derecho³.

"El desarrollo de estudios sociales sobre discapacidad ha sido muy escaso en nuestro país, pero también en el resto de América Latina y en España (...). Tal vez, esta ausencia de cuenta de las dificultades que implica la construcción de la discapacidad como objeto de estudio sociológico. Las mismas se plantean, en primer lugar, a la hora de definir qué entendemos por discapacidad. Se presentan al investigador "como combo cerrado" dos modelos explicativos contrapuestos: por un lado, el modelo rehabilitador, desde el cual la discapacidad es entendida como una enfermedad de un agente individual y tributaria de asistencia médica. Desde una perspectiva contrapuesta, el modelo social anglosajón comprende a la discapacidad como el resultado de la inadecuación de la estructura social a las necesidades de las personas con discapacidad, inadecuación que solo puede ser entendida al interior del sistema capitalista desde el cual los "discapacitados" constituyen mano de obra no productiva" (Ferrante, 2009, p. 18).

Ferrante (2009) cuestiona los sentidos de pretender abordar sociológicamente el concepto de discapacidad partiendo desde una mirada médica, resaltando la importancia de recordar que tenemos un "objeto de estudio que habla". Entre estas consideraciones, la autora afirma que aún quienes se han propuesto superar los reduccionismos de estas dos posturas reproducen el antagonismo sobre la naturaleza y la cultura. Para escapar a la postulación de instancias superadoras, refiere las problematizaciones de los modelos de abordaje desde campos disciplinares heterogéneos, para superar la "aceptación acrítica de estrechez analítica, que legitima prácticas normalizadoras” (Ferrante, 2009, p. 19).

En un trabajo que explora el vínculo entre los conceptos de discapacidad e inclusión desde las lógicas coloniales contemporáneas, Lorena Núñez Parra (2020) afirma que no se ha problematizado lo suficiente la forma en que se comprende epistémica y ontológicamente la discapacidad "como resultado de prácticas coloniales impregnadas de estructuras de poder" (Núñez, 2020, p. 63). Para esas lógicas: "este cuerpo mutilado, ingobernable, incivilizado, pero también expresión de resistencia pasó a ser a la vez un cuerpo improductivo" (Núñez, 2020, p. 64) y el concepto de inclusión (con sus correspondientes prácticas y políticas) pasó a ser indicador de su impuesta y autoritaria refuncionalización. Se vuelve evidente cómo aparecen las dinámicas, vinculadas: hay una clara intención de trabajar para que los estudiantes con discapacidad (como los demás estudiantes que van a la secundaria) puedan transformarse en ciudadanos útiles ${ }^{4}$.

3. Como ocurre en varios trabajos de investigación y otros espacios vinculados a la discapacidad.

4. Se reproduce el ideal capitalista de disciplinamiento social para que los estudiantes sean funcionales al mercado y a los parámetros hegemónicos de ser y de vivir (Vain, 2009). 


\section{Método}

Los estudios sobre trayectorias proponen líneas de tiempo, muestreo de las posibilidades, bifurcaciones, entre otras. En este sentido, se ha propuesto articular esta forma metodológica con el estudio de las biografías (Bolívar y Domingo, 2006), para poder volver evidente cómo la educación escolar formal hace parte de otros procesos vitales y se interdeterminan. Por supuesto que esto sucede con todas las personas, pero, específicamente en el caso del colectivo que analizamos, los sentidos y la experiencia de la discapacidad contribuyen a la construcción de ciertas particularidades.

Con respecto a las formas de construcción de la investigación narrativa, Bolívar (2002) afirma que los abordajes podrían formularse de acuerdo a dos posibilidades:

1. Análisis paradigmático de datos narrativos.

2. Análisis narrativo, propiamente dicho.

Para el análisis paradigmático de datos, se retoman las voces de los entrevistados, organizando comparaciones y generalizaciones que constituyen agrupaciones conceptuales para el análisis, pero la palabra que prima es la del investigador como autor del texto. Los datos se exponen para ilustrar (sic), un texto teórico y conceptual.

"En el segundo caso, se narra la historia respetando su totalidad en un relato nuevo, conjuntado (sic) por el investigador sin aspirar a una generalización. El análisis requiere que el investigador desarrolle una trama o argumento que le permita unir temporal o temáticamente los elementos, dando una respuesta comprensiva de por qué sucedió algo" (Bolívar, 2002, p. 13).

En esta investigación optamos por sostener los relatos completos, co-construidos con los actores, a fin de reivindicar las historias de los estudiantes como únicas, no generalizables, con sus dinámicas y elementos particulares. Esto también implica que el lector los conozca, otorgue sentido a esos elementos y se pueda evidenciar con claridad cuáles son las cuestiones que como investigadora elegí analizar y cuáles -aunque exceden los alcances del trabajo y no se retoman en este análisis- también las constituyen.

En todos los casos, se establece un contrato narrativo (Bolívar, 2012). Por las elecciones metodológicas de este trabajo, cada encuentro se configuró a partir de preguntas sobre las entrevistas anteriores y, en uno de los casos, una autobiografía. Las narrativas se presentan como resultado de ese proceso, entendiendo como eje, las trayectorias escolares. Es interesante ver las posibilidades de este tipo de investigaciones, según esa intención, teniendo en cuenta que podrían aparecer proyecciones hacia el punto de vista del investigador, por lo que es necesario atender al posible juego de distancias y proximidades entre la curiosidad del investigador y la abusiva puesta en escena de su visión u objetivos.

El desafío que se construye en este trabajo consiste (incluso actualmente, tras el proceso de tesis) en explicitar las historias de aquellos que no han sido conocidos y reconocidos formando parte de espacios de visibilización, no han influenciado con su trabajo en grandes movimientos, no son héroes. Son jóvenes estudiantes de escuelas secundarias transitando su cotidianeidad, con todo lo que eso implica, en las complejidades de las decisiones. Cuando se afirma que las decisiones son tomadas por otros se expone que el hecho de que los tres estudiantes están construyendo sus trayectorias escolares en instituciones que les 
permitirían, a futuro, alcanzar sus intenciones de ser médico, ser docente, ser científico, a partir de las oportunidades y elecciones que pudieron construir -en gran medida- por condiciones sociales de sus tutores (tener una madre docente), cuestiones de prestigio institucional (la escuela tiene estudiantes integrados con éxito), ofertas institucionales del contexto (la escuela de educación especial quedaba lejos).

Algunos autores como Muñiz, Argüello y Arduini, se ocuparon de la historia de esta perspectiva en América Latina. Reconocen su origen en la escuela de Chicago, a principios del siglo XX (Muñiz et al., 2014), admitiendo que desde los años 50, se intentó "conocer las problemáticas latinoamericanas con paradigmas, conceptos y métodos ajenos a nuestras realidades: la heterogeneidad cultural fue así comprendida desde valores, creencias y prácticas eurocéntricas” (Muñiz et al., 2014, p. 2). Por eso, la propuesta de los autores, desde los aportes de los estudios decoloniales, consistió en revisar algunos postulados metodológicos que han sido consolidados a lo largo de los años: "la concepción lineal de la temporalidad, las preguntas con que enfrentamos a las fuentes, y los distintos usos de la oralidad" (Muñiz et al., 2014).

Sobre el potencial de la investigación biográfica, inicialmente como dialógica y también como práctica decolonial, los autores siguen lo propuesto por Argüello (2012) y Birulés (1996) sobre la consolidación contemporánea de la perspectiva biográfica que plantea la construcción de un sujeto encarnado y situado que se aprecia tanto en su individualidad como en su sistema de relaciones, para estar atentos, específicamente a las "jerarquías epistémicas, espirituales, raciales/étnicas y de género/sexualidad desplegadas por la modernidad" (Castro-Gómez y Grosfoguel, 2007, pp. 13-14), que permiten la persistencia de estructuras colonizantes (Castro-Gómez y Grosfoguel, 2007) de los siglos XVI y XVII.

Se realizaron entrevistas biográficas que se complementaron con otras informaciones de contexto, como entrevistas a docentes, directivos, madres y padres. Además, se revisaron documentos institucionales y personales -obtenidos a partir del vínculo con las escuelas y las familias- a fin de completar los relatos.

Las etapas de la investigación consistieron en:

1. Definición de los objetivos, marcos de referencia y estado del arte.

2. Definición de la metodología y lecturas sobre producciones previas.

3. Realización de las entrevistas biográficas y entrevistas complementarias.

4. Sistematización y reorganización de los relatos.

5. Lectura y revisiones de los relatos por parte de los estudiantes participantes del estudio.

6. Análisis.

7. Escrituras y organización de la presentación final de la investigación.

Quienes han sido entrevistados para la realización de la reconstrucción biográfica fueron 3 estudiantes. Sus edades, años de nacimiento y cursos se encuentran descriptos en el apartado siguiente. 


\section{Resultados 5}

Por cuestiones de límites espaciales para la comunicación del trabajo, se sintetizarán en este apartado las reconstrucciones biográficas desde los hitos vitales mencionados por los tres estudiantes.

El estudiante 1 (en adelante, E1) nació en el año 2002 y en el momento de la entrevista (2018) cursaba el tercer año de secundaria. Nació con dextrocardia y varias complicaciones congénitas. En el año 2005 tuvo su primera cirugía de un riñón, mismo año en el que ingresó en la escolaridad inicial, razón por la que fue usuario de la educación hospitalaria. En el año 2006 pudo iniciar su escolaridad primaria en un colegio regular confesional pero las múltiples cirugías realizadas a más de mil kilómetros de su hogar implicaron volver al apoyo de educación hospitalaria, lo que permitió que en 2010 iniciara cuarto grado y cursara quinto. En 2011 comenzó sexto grado en una escuela estatal, pero debido a que en 2012 tuvo otras cirugías -a partir de las que se convirtió en usuario de una prótesis en una pierna y silla de ruedas- no pudo entrar en secundaria hasta 2016. Uno de los hitos que señala en su biografía es que, en el año 2015, ingresó como deportista en un equipo de deporte adaptado, actividad que le apasiona y que permitió que actualmente haya sido convocado por el seleccionador nacional.

Como cuestiones a destacar sobre la trayectoria escolar, se mencionará aquí que la escuela le parece significativa porque es un espacio de encuentro con amigos y diversión. A pesar de eso, afirma que se esfuerza por cumplir con las obligaciones que le permiten continuar con sus estudios y que las interrupciones fueron solamente por motivos de salud. Contaba en la entrevista que:

"Lo que pasa es que a mí nunca me gustó estudiar. Desde chico, con todas las materias me pasaba. Ni quería hablar en las clases. Ahora de grande, hay algunas materias que no me gustan mucho. Con las que me gustan, me pongo las pilas, pero las que no, me cuesta. Las maestras eran buenas, me acuerdo" (Entrevista biográfica, 2018).

El estudiante 2 (en adelante, E2) nació en el año 2000, en el momento de las entrevistas tenía 17 años y estaba en tercer año de secundaria. A los 14 se "quedó sordo" por un accidente automovilístico, razón por la cual fue derivado a una escuela de educación especial. El estudiante narra con enojo el momento, porque lo recuerda como el peor de su vida, y sintió que la escuela, en lugar de acompañarlo, lo excluyó. En la escuela de educación especial le enseñaron algunos contenidos para "nivelar" los conocimientos necesarios para el ingreso al nivel secundario (regular) y realizaron las gestiones para que fuera admitido en la única escuela secundaria de la comunidad rural donde vive.

Le gusta bastante estudiar, es un apasionado de la tecnología y tiene una beca de estudios para cursar un nivel básico de robótica. Una de sus actividades favoritas es fabricar robots con materiales reciclados.

La estudiante 3 (en adelante, E3) también nació en el 2000 y en el momento de la entrevista (2019) casi termina cuarto año. Cursó en una escuela de educación especial primer grado a los siete, segundo a los ocho y a los nueve el preparatorio para el ingreso a la escuela común. En 2009 intentó entrar en la escuela de música y la rechazaron, en 2013 ingresa finalmente en la escuela de música y, en 2015, en la educación secundaria.

5. En la investigación biográfica narrativa, las biografías no se consideran "resultados" sino oportunidades de aporte a partir de las reconstrucciones, también legítimas. Se consignan de esta manera por cuestiones de formalidad. 
Es la más crítica de los tres estudiantes con respecto a su trayectoria escolar y la única que expresó sentirse subestimada por lo que sabe y puede. Siente que las instituciones a las que concurrió no supieron comprender que ella no necesitaba esa gran cantidad de "apoyos" que recibió en la vida:

"Hoy siento que ir a la escuela común fue una decisión un poco impuesta. Me hacían ver que si vos lograbas irte era porque tu cabeza funcionaba. Si no, eras una mediocre, eras alguien que se quedó ahí porque no avanzó. Entonces yo decía que no soy así, que sé que puedo, que lo voy a lograr. Cuando yo estaba sola en el instituto, yo iba, hacía la tarea y podía jugar: después de la integración, ya no. Después de este momento tan abrumador y estresante, me acostumbré, y puedo decir que tuve una primaria linda. Mis compañeros comprendieron, en octubre más o menos de ese año, que yo era una niña como ellos, que podían compartir conmigo. Tenía dos compañeros varones que querían estar conmigo, hacíamos juntos los trabajos, se sentaban conmigo; eso estaba buenísimo, sobre todo esa época en la que en el colegio me miraban como un ser extraño, sobrenatural. Me han preguntado si yo era de la tierra o venía de otra parte [ríe]. Yo tenía que explicarles, porque los adultos no lo habían hecho". (Entrevista biográfica, 2019)

La E3 aclaró que no se siente inferior y que "percibe equidad". Menciona que no se siente limitada en cuanto a sus posibilidades, explicando sus logros (principalmente los escolares) con sus ansias de aprender. Cuando menciona que no le informaron sobre las "dificultades que podía llegar a tener", aclara que "no hubo un trauma", porque no reconoce otra forma de vivir o de ver. Siente que la discapacidad es una condición, aclarando la diferencia entre lo que percibe la sociedad, que usa el concepto como sinónimo de diferencia y limitaciones. Ella habla con seguridad de su potencial y quiere transmitir esa fuerza a todas las personas que la rodean. El asunto de las diferencias aparece en su relato vinculado también a cuestiones estéticas, para señalar que la discriminación puede encontrar un posible origen hasta en modos distintos de vivir. Ella reconoce que la discapacidad no es cuestión de patologías o capacidades.

Para el E1, conocer a otros jóvenes con discapacidad fue clave para valorar lo que él es. No ser el único, identificarse con pares, con quienes además comparte un espacio deportivo, significó la construcción de otros sentidos con respecto a la experiencia de la discapacidad.

El E2, vincula la discapacidad con "la mente", o con "andar mal de la cabeza". Su forma de referirse a ella deja ver que, por momentos, siente que no es un joven con discapacidad, sino, por el contrario, "solamente" es sordo. Afirma que "en el aprendizaje no tengo problemas, gracias a eso, pasé de curso. Fui a la escuela especial después del accidente y me dejaron ahí porque las otras no me recibían a mitad de año" (Entrevista biográfica, 2017).

En este caso, los tres se niegan a utilizar apoyos que resulten demasiado visibles o evidentes. Si en la escuela de E2 alguien comienza a usar la lengua de señas argentina (LSA) va a despertar la curiosidad de docentes y estudiantes, que se acercarían a mirar y preguntar (por lo que se pudo observar y consultar, siempre intenta pasar desapercibido); para E1 implicaría también ser mirado y podría desencadenar esas preguntas que no quiere que le hagan y, en el caso de E3, la evidencia de que tiene una discapacidad visual podría volver a presentarle situaciones de burla, como cuando -según su relato- fue víctima de las de sus compañeros.

E3 insiste con que no quiere "perder" más el tiempo, porque perdió dos años de su vida con apoyos que no necesitaba. En el caso de E2 también aparece una cuestión de pérdida con respecto a la escuela de educación especial. E3 entiende que la juventud es un momento para disfrutar ciertas cuestiones que des- 
pués, considera, desaparecerían o serían inaccesibles. E2 afirma que lo enviaron a la escuela de educación especial porque pensaron que estaba "totalmente discapacitado", pero que no era así, porque estaba "solamente sordo".

\section{Discusión}

Se ha señalado en investigaciones anteriores (Schewe, 2018) afines a la educación especial, que las prácticas cotidianas fueron apenas modificadas a partir de las propuestas de apoyos. Si se analiza, por ejemplo, cuáles son los sentidos actuales del trabajo en las escuelas de educación especial, nos encontramos con que siguen siendo espacios que contienen a grupos de estudiantes que -por motivos que determinan las instituciones- no estarían en condiciones de asistir a escuelas regulares. E2 afirma que lo enviaron a la escuela de educación especial porque pensaron que estaba "totalmente discapacitado", pero que no era así, porque estaba "solamente sordo". En este caso, es interesante el planteamiento de McRuer (2002) sobre la preferencia a las identidades capacitadas como aquello a lo que todas las personas aspiran que opera como una especie de sistema de capacidad corporal obligatoria compulsiva: los "discapacitados anormalizados" son otros.

Otros estudiantes atraviesan la totalidad de sus trayectorias en escuelas regulares, lo que también implica prever una serie de complejidades que han sido analizadas por varias autoras (Barrozo, 2017; Schwamberger et al., 2020; Bocchio et al., 2020) que se pueden visualizar como:

a. Si existen los recursos suficientes en cuanto a los apoyos necesarios, se garantiza la continuidad de las trayectorias. La mayoría de las escuelas públicas (podrían existir diferencias en algunos contextos con respecto a características como extensión geográfica, diversidad cultural y diferencias entre normativas jurisdiccionales) no cuentan con los recursos específicos en las instituciones, por lo que se recurre a los servicios de prestaciones sociales, convenios interinstitucionales o a solicitar a las familias de los estudiantes que cubran las demandas con recursos propios. Por eso, existen figuras como la del acompañante terapéutico ${ }^{6}$ y la de docentes de apoyo ${ }^{7}$, que son contratadas formal o informalmente.

b. Hay algunos casos en que no se considera necesario gestionar apoyos específicos. La cuestión es de qué forma y quiénes toman esa decisión porque, en ocasiones, como las escuelas no se ocupan de analizar cada caso, las trayectorias se ven interrumpidas y no se conocen los motivos. Por ejemplo, si los estudiantes se cansan de las incomodidades que genera la falta de apoyo y abandonan el curso, ese abandono se atribuye a la falta de interés del estudiante o al escaso apoyo de las familias, entre otras.

c. Se considera en este trabajo que la falta de los recursos específicos en las escuelas que analizamos es otra forma de violencia. Si bien las generalizaciones son arriesgadas, a más de 10 años de la implementación de marcos legales que promueven que los y las estudiantes con discapacidad lleven a cabo sus

6. Según Londero y Pacheco (2006), un acompañante terapéutico es un agente cualificado para ejercer un papel activo en el cotidiano de quienes sean usuarios de diferentes terapias. Los inicios del acompañamiento terapéutico como profesión se vinculan a los movimientos antimanicomiales de 1950.

7. Pueden ser contratados por las familias, las obras sociales o, en algunos casos, se destinan horas docentes de las escuelas de educación especial a esos fines. 
trayectorias escolares en todos los niveles y modalidades del sistema educativo argentino, continúan siendo excluidos/as, invisibilizados/as e infantilizados/as en las instituciones, lo que termina, en una gran cantidad de ocasiones, conduciendo al abandono.

Es pertinente destacar que, en ocasiones, las escuelas de educación especial son las que inician procesos de intercambio y formación de docentes de escuelas secundarias. Se conocen experiencias muy interesantes, pero también rechazos y discriminación hacia los estudiantes. En este sentido, es necesario mencionar que las condiciones de trabajo docente en estas escuelas continúan siendo modificadas a partir de prácticas que intentan aportar para garantizar el derecho a la educación a estudiantes con discapacidad, en las condiciones actuales:

\footnotetext{
"Uno de estos factores obstaculizantes que resulta necesario expresar tiene que ver con las condiciones de precarización, que se grafica en los largos recorridos que los y las docentes realizan entre las escuelas de distintos niveles educativos durante una jornada laboral, atravesando grandes distancias y utilizando distintos medios de transporte para llegar a tiempo a cumplir y garantizar los procesos educativos, entrando y saliendo por distintas aulas, interactuando con diversos actores educativos: directores, supervisores, docentes de áreas especiales, entre otros. Otra cuestión a señalar es que en las escuelas de educación secundaria el formato del saber disciplinar se diversifica a la vez que se complejiza; y se encuentra con la formación de los y las docentes de educación especial que acompañan el proceso educativo. Esto muchas veces resulta un obstáculo. Al respecto, una docente de apoyo a la inclusión lo expresa en una entrevista: 'yo me fijo las materias y le pregunto en qué tiene dificultad [...], pero la verdad que si me dice que no entiende Física yo me tengo que poner a estudiar, porque eso no lo vi en mi formación, y estudiar me lleva tiempo, sumado a los otros chicos que también realizo apoyo, así que es muy difícil' (Maestra de apoyo a la inclusión, 2017)". (Schwamberger et al., 2020, p. 53).
}

Una de las investigadoras destacadas en cuanto a estudios sobre maestras de apoyo a la inclusión (MAl) en Argentina es Cristina Pereyra (2013; 2015; 2017), quien analizó desde la etnografía una serie de espacios institucionales construidos, a fin de acompañar a estudiantes con discapacidad en sus trayectorias escolares. Sobre trayectorias escolares de estudiantes con discapacidad, desde lo biográfico narrativo, se reconocen como principales antecedentes:

1. El trabajo de Angélica Tobón (2019), La inclusión desde adentro y en primera persona: experiencias escolares de estudiantes con discapacidad en la educación secundaria en la ciudad de Buenos Aires. La autora analizó las biografías de seis estudiantes con discapacidad que cursan sus estudios en instituciones de educación regular, descubriendo interesantes formas de transitar ese nivel educativo a partir de tejidos vinculares de cada estudiante. Además de descubrir que a lo largo de la investigación algunos docentes e investigadores, "se mostraron reacios ante el hecho de entrevistar a estudiantes con discapacidad" (Tobón, 2019, p. 217), se hizo evidente en el trabajo la valoración de los procesos mencionados como de inclusión en tanto "posibilidad de estar juntos" (Tobón, 2019, p. 214) en la escuela. El trabajo presenta aportes en relación con la relevancia de las expectativas y el apoyo familiar, los mecanismos de exclusión que atraviesan los relatos y las contradicciones que genera para estas investigaciones el concepto de inclusión. En este caso, los 6 estudiantes con quienes se trabajó fueron varones porque la autora no pudo encontrar y vincularse con mujeres en esos espacios, lo que coincide con lo que evidencian las pocas estadísticas sobre la baja tasa de mujeres con discapacidad (Cobeñas, 2016) en las escuelas regulares.

En otro sentido, una invitación hacia nuevas continuidades del trabajo de Angélica Tobón se abre a partir de que algunos de estos estudiantes, aun habiendo llevado a término el último año y con esto la 
finalización de su trayectoria escolar, "no necesariamente tendrán como resultado la obtención del título secundario" (Tobón, 2019, p. 216) ${ }^{8}$, entendiendo que estas experiencias son consideradas nuevas para nuestros sistemas educativos y que queda un largo camino -hacia el reconocimiento de las trayectorias reales- por recorrer.

También, a partir del trabajo de Tobón, surge un cuestionamiento sobre las características que, por sugerencia de autores como Bolívar (2012), deberían tener las personas entrevistadas. Una de ellas es la de ser hábiles narradores/as, o tener una alta competencia narrativa, porque "un buen narrador facilita la entrevista, y relata mejor la historia que queremos" (Bolívar, 2012, p. 5). Las complejidades de estos procesos invitan a analizar que las intenciones de visibilizar las voces subalternizadas de algunos conviven con este tipo de sugerencias, que podrían continuar reproduciendo desigualdades y violencias sobre aquellos que no cumplen con el perfil del buen entrevistado/a, en el mismo sentido que las corponormatividades hegemónicas son excluyentes con las personas con discapacidad.

2. El trabajo de Anabel Moriña (2010) postula que las historias de los estudiantes con discapacidad se diferencian de las de otros estudiantes de su edad por los constantes cambios de escuelas, combinaciones con servicios de asistencias, situaciones de doble escolaridad, entre otras (por supuesto, salvando las distancias entre el contexto español y el argentino). La autora reconstruyó las biografías de nueve "personas con discapacidad derivada por dificultad intelectual, del habla o audición, de visión y de movimiento" (Moriña, 2010, p. 672), lo que coincide, en el caso de las últimas tres, con este trabajo.

De estos estudios, se destacan tramas como la siguiente:

"Siete de los nueve jóvenes han estudiado hasta la Educación Secundaria Obligatoria. De éstos, sólo dos han obtenido el título de Graduado Escolar. La excepción es una chica, quien en la actualidad está finalizando una carrera universitaria. La opción para muchos de estos jóvenes una vez finalizada o abandonada la Educación Secundaria ha sido la participación en Programas de Garantía Social (P.G.S.) con títulos de lo más diverso (peluquería, pintor empapelador, auxiliar de ayuda a domicilio, etc.) y cursos de formación ocupacional dirigidos a personas con discapacidad (auxiliar de oficina, frío industrial, etc.). Este es un hecho compartido por casi todos los chicos y chicas; comienzan en contextos educativos ordinarios, pero prefieren continuar en contextos especiales, como mejor alternativa para terminar sus estudios (en sus historias de vida, esta circunstancia se extiende a otros contextos, como el laboral o social)". (Moriña, 2010, p. 676).

Lo interesante de este tipo de estudios es que permiten desentrañar esas preferencias por los múltiples, complejos e interdeterminados procesos que podrían estar viviendo los sujetos, teniendo en cuenta -además-que se trata de procesos que son construidos desde conceptos polisémicos, como el de discapacidad.

Las experiencias analizadas en este trabajo difieren de varios estudios que plantean que los estudiantes con discapacidad prefieren refugiarse en contextos educativos específicos (Moriña, 2010) porque les resultan más acogedores: al contrario, en este caso, prefieren construir sus trayectorias en las escuelas comunes. Es relevante hablar de las preferencias de los estudiantes porque queda demostrado su consentimiento en la elección de las instituciones a las que quieren asistir; sin embargo, también forma parte de ese proceso la manera en que se construyen esas elecciones, por lo que se mencionaba anteriormente desde el planteamiento de McRuer (2002): ¿qué es lo deseable? y, específicamente, ¿qué es lo deseable para un joven adolescente?

8. Algunos estudiantes no obtienen dicho título y pasan a talleres donde las certificaciones son de oficios o se disponen a comenzar a trabajar, entre otras múltiples posibilidades. Las certificaciones continúan siendo objeto de discusión en Argentina ya que aún existen instituciones que se niegan a tramitar los títulos secundarios porque los estudiantes recibieron apoyos significativos o adaptaciones. 
En el caso de E3 el proceso fue diferente porque en el instituto al que asistía le habían hablado durante meses sobre lo que significaba ir a la escuela común, además de la propuesta de ciclo preparatorio, destinada a evitar un posible fracaso: era necesario anticipar hasta los contenidos que se trabajaría al año siguiente. Con emoción, narra el momento en que le dijeron "se está pensando en integrarte a vos", con todo lo que eso implicó finalmente.

Ella era la única de ese grupo que cumplía con requerimientos que no estaban explicitados con claridad pero que los demás compañeros del curso no cumplían. Los profesores les hacían sentir que "era un privilegio, lo máximo, el esplendor máximo al que un estudiante podría llegar en ese momento". Reconoce que podría resultar manipulable un niño/a que está "construyendo sueños", y que los planes de integración parecían una meta posible para todos, en ese momento.

Las relaciones entre las concepciones de los diferentes agentes escolares que participan en -y toman decisiones sobre- los procesos de escolarización y las condiciones materiales e institucionales de las escuelas en las que trabajan (Barrozo et al., 2017), resultan centrales para el análisis de las decisiones. Una de las formas que tomó el trabajo de los docentes de educación especial también fue realizar las gestiones vinculadas a las derivaciones a escuelas comunes.

Es pertinente observar cómo quienes comienzan desde pequeños sus trayectorias escolares en las escuelas de educación especial presentan estas idas y vueltas que mencionan las autoras con las escuelas comunes. Esto significa que, por diferentes motivos, hay estudiantes que, aunque intentan construir procesos que permitan la continuidad en la educación común, no lo logran. Entonces, dos preguntas interesantes son las que propone Pérez (2014, p. 162) "¿por qué la educación especial se presenta como lo no deseado? ¿Qué pasaría si uno pudiera elegir 'libremente' la escuela que desea para sus hijos e hijas, sin la imposición primera de un certificado médico que acredite una discapacidad?". La autora se pregunta también si la definición a priori del tipo de institución a la que debieran asistir los estudiantes a partir de diagnósticos no contribuye a perpetuarlos, condicionar las perspectivas y limitar autonomías y posibilidades, consolidando las desigualdades.

"Estoy loca, no me da la cabeza, estoy enfermo, mi mamá dice que yo no aprendo, las maestras de la otra escuela no me querían porque me portaba mal, no puedo escribir, nací con problemitas: son algunas de las afirmaciones de los estudiantes que formaron parte de estos procesos ante la pregunta sobre por qué asistían a la escuela de educación especial. Es frecuente avistar los prejuicios existentes sobre las discapacidades en las percepciones de niños y adultos, así como es preocupante el vínculo con la imposibilidad y la frustración”. (Schewe, 2020, p. 4)

Surge entonces la pregunta sobre la duda acerca de la posibilidad de que E3 hubiera tenido que aprender braille en una escuela común, teniendo en cuenta que, por ejemplo, para E2, no está aún disponible el apoyo en Lengua de Señas Argentina (LSA) en la escuela secundaria. Probablemente, por lo expresado hasta aquí, si se generan condiciones para que estén disponibles intervenciones profesionales que aborden esas especificidades (o para formar a quienes ya trabajen en ellas), se vuelvan posibles.

Una de las investigaciones que consideramos relevantes con respecto al currículum corresponde al trabajo de Calderón y Habegger (2012), en el que se plantea la lucha de una familia de un estudiante con síndrome de Down contra la escuela para demostrar que su hijo podía cursar toda su trayectoria escolar en la escuela común. Esto implicó romper con el orden natural que se ha construido hegemónicamente "de quienes nacieron para pensar, actuar socialmente, saber y poder sobre los que nacieron para las manualidades y cuya palabra no tiene lugar, no cuenta" (Greco, 2007, p. 96). 
El mexicano Quiróz, en 1992, analizó los sentidos del tiempo en la escuela secundaria. Si bien se trata de un estudio que corresponde a un contexto y un momento histórico bastante distinto al que se analiza en este trabajo, es interesante su propuesta de pensar que el tiempo tiene sentidos diferentes: para las autoridades, los docentes y los estudiantes. Los "atrasos" que mencionan E2 y E3 corresponderían a los tiempos de las autoridades y a los tiempos normativos del sistema educativo: a tiempos de control, vigilancia y deber ser. El autor menciona que para los estudiantes el tiempo se vincula a decisiones de la vida personal, a intereses extraescolares: televisión (podría tratarse actualmente de Internet, ver series, jugar en línea), amigos, deportes; lo que coincide con los sentidos que confiere E1 (Quiróz, 1992). También es necesario mencionar que estos tiempos pueden estar vinculados a las propuestas de los docentes.

Con respecto a estos actores del proceso (los docentes), una expresión que aparece con frecuencia en los relatos es que el estudiante con discapacidad: "no es para esa escuela". La expresión es un disfraz que deposita en el estudiante la falta, es él quien no correspondería a ese lugar. En cambio, podría estar señalando cuestiones institucionales, como la falta de recursos para recibir a todos los estudiantes, la necesidad de homogeneización de las infancias para lograr un ideal de disciplina, una forma estética, insuficiencias en la formación docente y prejuicios. Además, existiría cierta discrecionalidad en las decisiones que se toman, a partir de la interpretación de situaciones particulares del estudiante, sin indagar en las complejidades de las manifestaciones. En esos casos, los adultos que toman las decisiones suponen que responsabilizarse implica derivar, entrevistar, medicar y conjeturar, mientras crean incertidumbre y temores para estudiantes y sus familias: no les explican con claridad y no les permiten participar en las decisiones que se toman.

Para el caso de la construcción de la discapacidad, podríamos afirmar que también se trata de una historia de disputas por la legitimidad de ciertos saberes: el religioso, el asistencialista, el rehabilitador, el médico, el del derecho, el de los educadores. Estos saberes dan lugar a prácticas y al sostenimiento o creación de espacios que, por momentos, hasta se convierten en grandes negocios, como los que se crearon desde la patologización y la medicalización de las infancias con la industria farmacológica. La pregunta entonces, a partir de lo dicho en este trabajo sobre los lugares de enunciación y los estudios crip, es ¿estaremos -al fin- ante el surgimiento del reconocimiento de los propios discursos de las personas con discapacidad? ¿Puede la escuela secundaria ofrecer a los jóvenes un espacio para transgredir las formas hegemónicas de vivir sin ser castigados? "¿Presenta la escuela las características que la hagan factible de abarcar exitosamente a todas las clases sociales, a todas las culturas y trayectorias escolares?" (Southwell, 2020, p. 3). También es parte de estos procesos, la responsabilidad que tienen las escuelas sobre la confianza plena de los estudiantes en su esfuerzo individual y sobre el hecho de que no hayan nombrado espacios colectivos de reivindicaciones y resistencias, exceptuando alguna referencia a trabajos grupales, amistad y acciones solidarias, que también terminan siendo individuales.

\section{Conclusiones}

Lo dicho confirma la certeza de que las personas con discapacidad tienen que formar parte de los espacios de construcción de saberes sobre ellos, para no reproducir las sospechas y generar alternativas pertinentes para pluralizar las educaciones. 
Es necesario mencionar que -si bien E2 y E3 manifiestan una especie de rechazo a la educación especial y consideran sus trayectorias en las escuelas específicas como pérdida de tiempo- en ese espacio construyeron dos cuestiones que fueron fundamentales para sus continuidades: E3 aprendió el braille (que le permite la escritura que elige hasta la actualidad) y E2 pudo acceder a la secundaria por las gestiones que hicieron las docentes de una escuela de educación especial. En el caso de E1, las continuidades fueron dadas en el momento en que la educación hospitalaria era parte de la educación especial.

Aún falta un camino por recorrer en cuanto a la accesibilidad en las escuelas secundarias, lo que no responde solamente a la cuestión de los recursos. Muchas de las condiciones para las continuidades fueron creadas gracias a las voluntades individuales de docentes y estudiantes. Docentes que invierten horas que no están reconocidas económicamente para discutir sobre los procesos, que buscan en Internet al azar información sobre discapacidades; estudiantes que diseñan sus propios apoyos; directivos que trasladan a estudiantes en sus vehículos particulares durante cientos de kilómetros para generar participación en otros espacios.

María da Conceição Passeggi (2015, p. 71) decía: "la cuestión es saber cómo llegar a la experiencia humana y cómo ella puede transformarse en conocimiento científico y emancipador: las narrativas (...) parecen ser una vía de acceso privilegiado a lo humano y a otra forma de hacer ciencia". Hacia ahí se orientó esta búsqueda, por lo que, finalmente, este trabajo es un aporte para continuar construyendo la perspectiva de los estudios críticos en discapacidad en Latinoamérica, a partir de prácticas de investigación que se manifiesten en contra de todas las acciones desde las ciencias que resulten invasivas, violentas y patologizantes; sistematizar experiencias que resultan novedosas para el sistema educativo argentino y se construyen con el compromiso y el esfuerzo de quienes se encuentran trabajando en las instituciones y visibilizar experiencias creativas y potentes en contextos asociados a prejuicios.

Se considera no solo pertinente, sino también enriquecedora, la elección metodológica de la tesis. Una gran cantidad de investigaciones contemporáneas sobre la temática se han ocupado de analizar cuestiones legales o de confirmar que los derechos de los estudiantes con discapacidad están siendo vulnerados, pero centrándose en la aplicación de normativas. Este trabajo permitió mirar la forma en que, en los procesos vitales, el cumplimiento de esas normativas (y todas las decisiones que se vinculan) van generando sentidos y procesos que permiten continuidades que son muy complejas, porque se entretejen a cuestiones familiares y contextuales. 


\section{Referencias bibliográficas}

Argentina. Ley $N^{\circ}$ 26.206, de 14 de diciembre de 2006, de Educación Nacional. http://servicios.infoleg.gob.ar/ infolegInternet/anexos/120000-124999/123542/norma.htm.

Argüello, A. (2012). Entre el tiempo y el relato: consideraciones epistemológicas en torno a la perspectiva biográfica en la investigación social y educativa. CPU-e: Revista de Investigación Educativa, (15), pp. 27-47. https:// www.redalyc.org/pdf/2831/283123579002.pdf.

Barrozo, N. et al. (2017). Trayectorias escolares de personas con discapacidad en la educación secundaria en Argentina. En M. Rodríguez (Comp.), Prácticas innovadoras inclusivas: retos y oportunidades (pp. 1595-1063). Servicio de Publicaciones de la Universidad de Oviedo. http://digibuo.uniovi.es/dspace/bitstream/10651/50310/4/ TrayectoriasEscolares.pdf.

Birulés, F. (1996). Del sujeto a la subjetividad. Duro deseo de durar. En M. Cruz (Comp.), Tiempo de subjetividad (pp. 223-234). Paidós.

Bocchio, M. et al. (2020). Inclusión gerenciada y escolarizaciones low cost. Una analítica de episodios en escuelas estatales del sur global". Revista latinoamericana de educación inclusiva, 14(2), pp. 177-190. https://scielo. conicyt.cl/scielo.php?pid=S0718-73782020000200177\&script=sci_arttext\&tlng=en.

Bolívar, A. (2002). ¿De nobis ipsis silemus?: epistemología de la investigación biográfico-narrativa en educación. Revista Electrónica de Investigación Educativa, 4(1). http://www.scielo.org.mx/scielo.php?script=sci_arttext\& pid=S1607-40412002000100003.

Bolívar, A. (2012). Metodología de la investigación biográfico-narrativa: recogida y análisis de datos. Dimensões epistemológicas e metodológicas da pesquisa (auto) biográfica. EDIPUCRS.

Bolívar, A. y Domingo, J. (2006). La investigación biográfica y narrativa en Iberoamérica: campos de desarrollo y estado actual. Forum: Qualitative Social Research, 7(4). http://dx.doi.org/10.17169/fqs-7.4.161.

Briscioli, B. (2013). Tendencias y puntos críticos en las trayectorias escolares de estudiantes de Escuelas de Reingreso de la Ciudad de Buenos Aires. Una indagación sobre las condiciones de escolarización en la construcción de las trayectorias escolares [Tesis de Doctorado no publicada]. Universidad Nacional de Entre Ríos.

Calderón, I. y Habegger, S. (2012). Educación, hándicap e inclusión. Una lucha familiar contra una escuela excluyente. Octaedro.

Castro-Gómez, S. y Grosfoguel, R. (2007). El giro decolonial. Reflexiones para una diversidad epistémica más allá del capitalismo global. Siglo del Hombre Editores.

Cheli, M. V. (2013). La construcción histórica de las clasificaciones y jerarquización de la infancia en el proceso de escolarización: un análisis en la provincia de Buenos Aires (1880-1952) [Tesis de Maestría]. Universidad Nacional de La Plata. http://www.memoria.fahce.unlp.edu.ar/library?a=d\&c=tesis\&d=Jte1260.

Cobeñas, P. (2016). Jóvenes mujeres con discapacidad en escuelas públicas de la provincia de Buenos Aires: problematizando los procesos de inclusión y exclusión educativa. [Tesis doctoral]. Universidad Nacional de La Plata. https://memoria.fahce.unlp.edu.ar/library?a=d\&c=tesis\&d=Jte1213.

Danel, P. M. (2018). Discapacidad: tensiones entre la opresión y las prácticas liberadoras. Trabajo Social Global, 8. 
Ferrante, C. (2009). Cuerpo, discapacidad y violencia simbólica. Un acercamiento a la experiencia de la discapacidad motriz como relación de dominación encarnada. Onteaiken. Boletín sobre prácticas y experiencias educativas, 8, pp. 17-34. http://www.repositoriocdpd.net:8080/bitstream/handle/123456789/352/Art_FerranteC_CuerpoDiscapacidadViolencia_2009.pdf?sequence=1.

Greco, M. B. (2007). La autoridad (pedagógica) en cuestión. Enfoques y perspectivas. Homo Sapiens.

Londero, I. y Pacheco, J. T. B. (2006). ¿Por qué encaminar al acompañante terapéutico? una discusión considerando la perspectiva de psicólogos y psiquiatras. Psicologia em estudo, 11(2), pp. 259-267. https://siteat.net/ igor/.

McRuer, R. (2002). Compulsory able-bodiedness and queer/disabled existence. En S. L. Snyder et al. (Eds.), Disability studies: Enabling the humanities (pp. 88-99). Modern Language Association.

Moriña, A. (2010). Vulnerables al silencio. Historias escolares de jóvenes con discapacidad. Revista de Educación, 353, pp. 667-690. http://www.revistaeducacion.educacion.es/re353/re353_25.pdf.

Muñiz, L. et al. (27-29 de agosto de 2014). Emergencias continentales de la perspectiva biográfica: reflexiones epistemológicas y metodológicas desde América Latina [Actas de congreso]. IV Encuentro Latinoamericano de Metodología de las Ciencias Sociales. La investigación social ante desafíos transnacionales: procesos globales, problemáticas emergentes y perspectivas de integración regional, Heredia, Costa Rica.

Núñez, L. (2020). Discapacidad y trabajo: la individualización de la inclusión bajo lógicas coloniales contemporáneas. Nómadas, 52, pp. 61-79. https://dx.doi.org/10.30578/nomadas.n52a4.

Passeggi, M. C. (2015). Narrativa, experiencia y reflexión auto-biográfica: por una epistemología del sur en Educación. En G. J. Murillo (Comp.). Narrativas de experiencia en educación y pedagogía de la memoria (pp. 69-88). Editorial de la Facultad de Filosofía y Letras Universidad de Buenos Aires, UdeA, CLACSO. http:// biblioteca.clacso.edu.ar/clacso/se/20160824025815/Narrativas-de-experiencias-de-educacion-y-pedagogia. pdf.

Pereyra, C. (2013). Procesos de derivación de educación común a educación especial de niños y niñas que habitan en contextos de pobreza. Textos y contextos desde el sur; 1(1), pp. 15-28. http://www.revistas.unp.edu.ar/ index.php/textosycontextos/article/view/10.

Pereyra, C. (2015). La configuración de circuitos escolares diferenciados en las derivaciones a educación especial de niños/as en contextos de pobreza. Revista RUEDES, 4(6), pp. 58-73. http://www.bdigital.uncuyo.edu.ar/ app/navegador/?idobjeto=6906.

Pereyra, C. (2017). El espacio de los viernes: sentidos y saberes docentes en torno a las prácticas de las maestras de apoyo a la inclusión. http://rephip.unr.edu.ar/bitstream/handle/2133/14789/El\%20espacio\%20de\%20 los\%20viernes\%20.pdf? sequence=3\&isAllowed=y.

Pérez, A. (2014). Educación especial y alteridad: en busca de lo común. Revista Latinoamericana de Educación Inclusiva, 8(1), pp. 153-169. http://www.rinace.net/rlei/numeros/vol8-num1/art10.pdf.

Quiróz, R. (1992). El tiempo cotidiano en la escuela secundaria. Nueva Antropología, 12(42), pp. 89-100. https:// www.redalyc.org/pdf/159/15904207.pdf.

Schewe, L. (2011). La educación especial desde una perspectiva sociocomunitaria: una experiencia sobre la relocalización en la escuela especial № 45". Revista RUEDES, 1(2), pp. 133- 138. https://bdigital.uncu.edu.ar/ objetos_digitales/3948/scheweruedes2.pdf. 
Schewe, L. (2018). Políticas de inclusión educativa de personas con discapacidad. Continuidades y discontinuidades en los procesos para su implementación [Tesis de maestría no publicada]. Universidad Nacional de Quilmes.

Schewe, L. (2020). Derivaciones y cambios de escuela como formas de violencias sobre las infancias que se discapacitan. En I. Ramírez et al. (Comps.), Infancias, cuerpos y discapacidad III: diálogos e intersecciones (pp. 61-72). Gaviota del Sur.

Schewe, L. (2021). Trayectorias escolares de estudiantes con discapacidad: logros, obstáculos y desafíos en la educación secundaria [Tesis doctoral]. Universidad Nacional de La Plata. http://sedici.unlp.edu.ar/handle/10915/117194.

Schwamberger, C. et al. (2020). Educación especial, trabajo docente e inclusión en Argentina: tensiones, eufemismos y contradicciones. Revista Senderos Pedagógicos, 11(11), pp. 45-57. https://doi.org/10.53995/sp. v11i11.932.

Southwell, M. (2020). La escuela secundaria frente al desafío de la universalización: debates y experiencias en Argentina. Education Policy Analysis Archives, 28(39), pp. 1-22. https://d1wqtxts1xzle7.cloudfront.net/62390519/ EPAA_sobre_obligatoriedad_de_secundaria.

Terigi, F. (28-30 de mayo de 2007). Los desafíos que plantean las trayectorias escolares [Conferencia]. III Foro Latinoamericano de Educación Jóvenes y docentes. La escuela secundaria en el mundo de hoy, Buenos Aires. http://www.ieo.edu.ar/promedu/trayescolar/desafios.pdf.

Tobón, A. M. (2019). La inclusión desde adentro y en primera persona: experiencias escolares de estudiantes con discapacidad en la educación secundaria en la ciudad de Buenos Aires [Tesis de Maestría]. Facultad Latinoamericana de Ciencias Sociales. http://dspace.eastus2.cloudapp.azure.com/handle/123456789/84.

Torres, A. A. et al (2019). Actitudes hacia la discapacidad en una universidad mexicana. Revista Brasileira de Educação, 24. https://www.redalyc.org/articulo.oa?id=27559571018.

Vain, P. D. (2009). Escuela, Estado y familia. Un pacto por redefinir. Educación, lenguaje y sociedad, 6(6), pp. 329344. http://www.biblioteca.unlpam.edu.ar/pubpdf/ieles/n06a17vain.pdf. 